\section{Was bringt die Interleukin-5-Hemmung bei eosinophiler Ösophagitis?}

\section{Die Therapie der eosinophilen Ösophagitis ist vor allem bei Kindern und Jugendlichen problematisch. Denn die üblichen Glukokortikoide können unter anderem das Wachstum verzögern. US-Forscher haben nun versucht herauszufinden, ob den Betroffenen mit dem Interleukin-5-Hemmer Reslizumab zu helfen ist. Ergebnis: teils, teils.}

E rkenntnisse aus Tierversuchen legen nahe, dass Interleukin 5 (IL-5) die eosinophile Entzündung im Ösophagus befeuert. Reslizumab ist ein monoklonaler Antikörper, der in der Lage ist, IL-5 zu neutralisieren. Daher könnte sich mit der Substanz auch der Verlauf der eosinophilen Ösophagitis beeinflussen lassen.

An einer entsprechenden randomisierten, doppelt verblindeten und placebokontrollierten Studie beteiligten sich 226 Patienten im Alter von fünf bis 18 Jahren. Rund 90\% von ihnen wiesen klinisch mäßige bis sehr schwere Symptome auf, wie etwa Schmerzen, Erbrechen oder Schluckbeschwerden. Im Median waren in den Gewebeproben der Speiseröhre 80 intraepitheliale Eosinophile pro Hauptgesichtsfeld (400-fache Vergrößerung, 0,3 $\mathrm{mm}^{2}$ ) zu finden. Zu Beginn der Studie sowie nach vier, acht und zwölf Wochen erhielten die Probanden 1, 2 oder $3 \mathrm{mg} / \mathrm{kg}$ Reslizumab oder eine Placebolösung als Infusion. Nach 15 Wochen wurden die Gewebseosinophilen erneut gezählt. Außerdem wurde der klinische Befund erhoben und anhand des „Physician's Eosinophilic Esophagitis Global Assessment" einem von fünf Graden zugeordnet.

Tatsächlich hatte die Zahl der Eosinophilen bei jenen Probanden, die den IL5-Hemmer infundiert bekommen hatten, im Vergleich zu Placebo signifikant abgenommen. Die Reduktion betrug 59\% in der 1-mg-, 67\% in der 2-mg- und 64\% in der 3-mg-Gruppe. Auch klinisch ergaben sich Verbesserungen: Hatten zu Studienbeginn nur knapp 10\% der Teilnehmer keine oder leichte Beschwerden, waren es nun $56,4 \%$ (1 mg), 63,2\% (2 mg), 64,9\% (3 mg) bzw. 71,9\% (Placebo). Die Verumsubstanz schnitt hier aber nicht signifikant besser ab als Placebo.

Fazit: Reslizumab wirkt gegen eosinophile Ösophagitis von Kindern und Jugendlichen - zumindest unter dem Mikroskop. Klinisch bringt eine zwölfwöchige Intervalltherapie zunächst keine Vorteile gegenüber Placebo. Ob eine längerfristige Therapie besser abschneidet, wird zurzeit untersucht.

Dr. Robert Bublak

Spergel JM et al. Reslizumab in children and adolescents with eosinophilic esophagitis: Results of a double-blind, randomized, placebo-controlled trial. J Allergy Clin Immunol 2012; 129: 456-63

\section{Insektengift: Vier Faktoren zeigen erhöhte Schockgefahr an}

Welche Parameter im Zusammenhang mit schwerwiegenden anaphylaktischen Reaktionen nach Insektenstichen auftreten, haben Dr. Johanna Stoevesandt und ihre Kollegen von der Universitätsklinik in Würzburg untersucht. Dabei haben sie vier Faktoren ermittelt, mit denen Ärzte eine hohe Schockgefahr erkennen können.

$S^{\mathrm{i}}$ ie sichteten die Daten von 657 Insektengiftallergikern, die sich zwischen 2003 und 2010 in der Würzburger Klinik vorgestellt und die Kriterien für eine Immuntherapie erfüllt hatten. 172 Patienten (26\%) hatten anamnestisch eine schwere anaphylaktische Reaktion erlitten, 277 Patienten (42\%) eine mittelschwere und 208 Patienten (32\%) eine milde.

Die Allergologen suchten in den Krankenakten zum einen nach patientenspezifischen Risikofaktoren wie Alter, Geschlecht, kardiopulmonalen Erkrankungen und kardiovaskulären Medikamenten. Zum anderen berücksichtigten sie Details im Zusammenhang mit der anaphylaktische Reaktion: Von welchem
Insekt und wohin war der Patient gestochen worden? Wie viel Zeit war zwischen Stich und ersten Symptomen vergangen? Traten Hautveränderungen auf? Bei einigen Patienten mit mittelgradigen bis schweren Anaphylaxien war zudem der Basalwert der Serumtryptase (BST) gemessen worden.

Ergebnis: Bei vier Faktoren war danach das Risiko einer schweren Anaphylaxie signifikant erhöht: Serumtryptase-Werte über 11,4 $\mu \mathrm{g} / \mathrm{l}$, Fehlen einer Hautreaktion, hohes Alter und unmittelbare Symptome (weniger als fünf Minuten zwischen Stich und allergischer Reaktion).

Die anderen untersuchten Parameter blieben hingegen für das Schockrisiko ohne Bedeutung. So hatten Insektengiftallergiker, die an kardiopulmonalen Erkrankungen litten oder Betablocker, ACEHemmer oder beides einnahmen, nicht häufiger schwere Anaphylaxien als Patienten ohne diese Faktoren.

Fazit: Um bei Insektengiftallergie das Risiko einer schweren Anaphylaxie abschätzen zu können, ist auf vier Faktoren zu achten: auf das Alter der Betroffenen, auf eine mögliche Mastozytose, die sich in hohen BST-Werten niederschlägt, eine fehlende Hautreaktion sowie eine kurze Zeitspanne zwischen Stich und Anaphylaxie. Eine kardiovaskuläre Medikation hingegen könnte als Risikofaktor bislang überbewertet worden sein.

Dr. Dagmar Kraus

Stoevesandt J et al. Over- and underestimated parameters in severe Hymenoptera venom-induced anaphylaxis: Cardiovascular medication and absence of urticaria/angioedema. J Allergy Clin Immunol 2012; 130 : 698-704 